doi:10.13108/2020-12-1-30

\title{
EQUIVALENCE CRITERION FOR TWO ASYMPTOTIC FORMULAE
}

\author{
Kh.K. ISHKIN, R.I. MARVANOV
}

\begin{abstract}
We study the equivalence conditions of two asymptotic formulae for an arbitrary non-decreasing unbounded sequence $\left\{\lambda_{n}\right\}$. We show that if $g$ is a non-decreasing and unbounded at infinity function, $\left\{f_{n}\right\}$ is a non-decreasing sequence asymptotically inverse to the function $g$, then for each sequence of real numbers $\lambda_{n}$ satisfying an asymptotic estimate $\lambda_{n} \sim f_{n}, n \rightarrow+\infty$, the estimate $N(\lambda) \sim g(\lambda), \lambda \rightarrow+\infty$, holds if and only if $g$ is a pseudo-regularly varying function (PRV-function). We find a necessary and sufficient condition for the non-decreasing sequence $\left\{f_{n}\right\}$ and the function $g$, under which the second formula implies the first one. Employing this criterion, we find a non-trivial class of perturbations preserving the asymptotics of the spectrum of an arbitrary closed densely defined in a separable Hilbert space operator possessing at least one ray of the best decay of the resolvent. This result is the first generalization of the a known Keldysh theorem to the case of operators not close to self-adjoint or normal, whose spectra can strongly vary under small perturbations. We also obtain sufficient conditions for a potential ensuring that the spectrum of the Strum-Liouville operator on a curve has the same asymptotics as for the potential with finitely many poles in a convex hull of the curve obeying the trivial monodromy condition. These sufficient conditions are close to necessary ones.
\end{abstract}

Keywords: asymptotic equivalence, functions preserving equivalence, pseudo-regularly varying (PRV) functions, non-self-adjoint operators, Keldysh theorem, spectrum localization, potentials with trivial monodromy.

Mathematics Subject Classification: 34D05, 35P20, 60F17

\section{INTRODUCTION}

To find an asymptotics of some sequence $\left\{\lambda_{n}\right\}_{n=1}^{\infty}$ means to find a sequence $\left\{f_{n}\right\}$ with properties known according the context such that the relation

$$
\lambda_{n} \sim f_{n}, \quad n \rightarrow+\infty,
$$

holds. Hereinafter the writing $f(x) \sim g(x), x \rightarrow x_{0}, x \in D$, is treated as an asymptotic identity, equivalence, for the functions $g$ and $f$ defined on the set $D$ with a limiting point $x_{0}$ :

$$
g(x)=f(x)(1+\alpha(x)), \quad x \in D, \quad \lim _{x \rightarrow x_{0}} \alpha(x)=0 .
$$

Sometimes formula (1) can be specified. For instance, let $L$ be an operator in $L^{2}(0, \pi)$ generated by the differential expression $-y^{\prime \prime}+q y$, where $q \in W_{2}^{m}[0, \pi]$, and by the boundary conditions

Kh.K. Ishinin, R.I. Marvanov, Equivalence CRIterion for two asymptotic Formulae.

(C)IshKin Kh.K., Marvanov R.I. 2020.

The research is financially supported by grant of Russian Science Foundation (project no. 18-11-00002).

Submitted June 20, 2019. 
$y(0)=y(\pi)=0$. Let $\left\{\lambda_{n}\right\}$ be the eigenvalues of $L$ taken counting algebraic multiplicities in the order of ascending absolute values. Then [2, Ch. I, Sect. 5]):

$$
\lambda_{n}-\left(n^{2}+\sum_{j=1}^{[m+1 / 2]} c_{j} n^{-2 j}\right)=n^{-m} \alpha_{n}, \quad c_{j}=\text { const }, \quad\left\{\alpha_{n}\right\} \in l^{2} .
$$

However, in the problems, in which one has to deal with the sequences, one employs the formula

$$
N(\lambda) \sim g(\lambda), \quad \lambda \rightarrow+\infty,
$$

where

$$
N(\lambda)=\sum_{\lambda_{n}<\lambda} 1
$$

is the counting function of $\left\{\lambda_{n}\right\}$. The motivation for this can be various; sometimes the choice of formula (2) is not because of employed methods and approaches, but due to deeper reasons coming from the matter of the studied problem. As a demonstration, we consider the following example.

Example 1. Let $G$ be a bounded domain in $\mathbb{R}^{m},-\Delta_{D}^{G}$ be the Dirichlet Laplacian in the domain $G$, which is the self-adjoint operator in $L^{2}(G, d x)$ associated with the quadratic form being the closure of the form

$$
q(f, g)=\int_{G} \nabla f \bar{\nabla} g d x, \quad f, g \in C_{0}^{\infty}(G) .
$$

Let $\left\{\lambda_{n}\right\}$ be the eigenvalues of the operator $A$ taken in the ascending order counting the multiplicities. If $G$ has a Jordan volume, then [3, Ch. XIII, Sect. 15]

$$
N(\lambda) \sim(2 \pi)^{-m} \tau_{m} W_{m}(G) \lambda^{\frac{m}{2}}, \quad \lambda \rightarrow+\infty,
$$

where $W_{k}$ is a $k$-dimensional Jordan volume. Under additional assumptions on the domain $G$ and its boundary $\Gamma$, formula (3) can be specified and the next-to-leading term can be found [4, Sects. 17.5, 24.7], [5]. This term is expressed via the $(m-1)$-dimensional measure of $\Gamma$.

We also note that formula (3) goes back to a known work by G. Weyl [6], which confirmed the Lorentz and Jeans conjecture on determining the volume of the domain $G$ by the spectrum of the Laplacian $-\Delta^{G}$. Later there was found a series of other geometric characteristics of the domain $G$ determined by the asymptotics of $N(\lambda)$ [7].

Example 1 shows how natural can be the choice of formula (2). Of course, at that one assumes that the information on the values of the function $g$ gives some information on the sequence $\left\{f_{n}\right\}$. This assumption is justified, for instance, in the case, when the sequence $\left\{\lambda_{n}\right\}$ is strictly monotone and the function $g$ preserves the asymptotic equivalence of sequences, that is, for all infinitely large sequences $\left\{x_{n}\right\}$ and $\left\{y_{n}\right\}$ the relation

$$
x_{n} \sim y_{n}, \quad n \rightarrow+\infty \Rightarrow g\left(x_{n}\right) \sim g\left(y_{n}\right), \quad n \rightarrow+\infty,
$$

holds true. Indeed, if $\left\{\lambda_{n}\right\}$ is increasing, then $N\left(\lambda_{n}\right)=n-1$, and by formulae (2) and (4) we have

$$
g\left(f_{n}\right) \sim n, \quad n \rightarrow \infty .
$$

If we also assume that the function $g$ is continuous and increases on $[A,+\infty)$ and the inverse function $g^{-1}$ also preserves the asymptotics, then by relation (5) we obtain $f_{n} \sim g^{-1}(n)$ as $n \rightarrow+\infty$.

In what follows, in Lemma 1, we shall show that if the sequence $\left\{f_{n}\right\}$ and the function $g$ are non-decreasing, then condition (5) is necessary (but is not sufficient!) in order to for each non-decreasing sequence $\left\{\lambda_{n}\right\}$ obeying estimate (1), estimate (2) is to be satisfied. 
The aim of the present note is to find necessary and sufficient condition for the function $g$ or sequence $\left\{f_{n}\right\}$ ensuring that for each non-decreasing sequence $\left\{\lambda_{n}\right\}$ obeying one of formulae (1) and (2), the other formula holds true.

Before we formulate the results, we mention that the class of the functions preserving asymptotic equivalence (4) is well-known. For further purposes, it is convenient for us to formulate a criterion obtained by V. V. Buldygin, O. I. Klesov, J. G. Steinebach in [8].

Theorem BKS. Let a function $g$ be measurable on $[A,+\infty)$ and $\lim _{x \rightarrow+\infty} g(x)=+\infty$. Then the following statements are equivalent:

1) The function $g$ preserves an asymptotic equivalence of sequences.

2) The function $g$ preserves asymptotic equivalence of continuous functions, that is, $g(u(t)) \sim$ $g(v(t)), t \rightarrow+\infty$, for all functions $u, v$ continuous on some interval $(B,+\infty)$, asymptotically equivalent on $+\infty$ and satisfying the condition

$$
\lim _{t \rightarrow+\infty} u(t)=\lim _{t \rightarrow+\infty} v(t)=+\infty .
$$

3) The function $g$ satisfies the condition

$$
\lim _{\delta \rightarrow 1+0} \limsup _{x \rightarrow+\infty} \frac{g(\delta x)}{g(x)}=1 .
$$

It follows from this theorem that if the function $g$ is continuous on $[A,+\infty)$, increases, is unbounded, and $f_{n}=g^{-1}(n)$, then condition (6) is sufficient to ensure that each increasing sequence $\left\{\lambda_{n}\right\}$ with asymptotics (1) satisfies also (2). Indeed, under the maid assumption,

$$
N(\lambda)=n, \quad g\left(\lambda_{n}\right)<g(\lambda)<g\left(\lambda_{n+1}\right) \quad \text { on } \quad\left(\lambda_{n}, \lambda_{n+1}\right]
$$

and $g\left(\lambda_{n}\right) \sim n, n \rightarrow+\infty$ by (1) and Statement 1) of the theorem. This implies (2).

Remark 1. The functions obeying condition (6) are called PRV-functions (pseudo-regularly varying). In view of numerous applications, especially in the theory of probability, the PRVfunctions are studied quite well, see [9] and the references therein. PRV-functions are natural generalizations of $R V$-class of regularly varying functions introduced in 1930 by Karamata in his fundamental work [10]. The results by Karamata together with further extensions and generalization turned out to be very fruitful for various fields in mathematics, see [11], [12].

\section{Formulation of MAin Results}

Hereafter $\left\{\lambda_{n}\right\}_{n=1}^{\infty}$ stands for an arbitrary real non-decreasing sequence, $S^{\infty}$ is the set of nondecreasing unbounded sequences, $F^{\infty}$ is the set of functions defined on some interval $(A,+\infty)$ depending on the choice of the function; these functions are finite, non-decreasing and unbounded.

We begin with a simple statement.

Lemma 1. Let $\left\{f_{n}\right\} \in S^{\infty}, g \in F^{\infty}$ be such that for each sequence $\left\{\lambda_{n}\right\}$ obeying (1), estimate (2) is satisfied as well. Then (5) holds true.

The main result of the paper is formulated in the following two theorems.

Theorem 1. Let $\left\{f_{n}\right\} \in S^{\infty}, g \in F^{\infty}$. If conditions (5) and (6) are satisfied, then for each sequence $\left\{\lambda_{n}\right\}$ satisfying (1) estimate (2) holds true.

And vice versa, if for each sequence $\left\{\lambda_{n}\right\}$ satisfying (1) estimate (2), then $\left\{f_{n}\right\}$ and $g$ satisfy (5) and (6). 
The next theorem is about the conditions, under which for each sequence in $S^{\infty}$ the implication holds: $(2) \Rightarrow(1)$. But before we should check the existence of at least one sequence in $S^{\infty}$ satisfying (2). We observe that under the assumptions of Theorem 1 such issue does not arise. The following lemma holds true.

Lemma 2. There exists at least one sequence $\left\{\lambda_{n}\right\} \in S^{\infty}$ satisfying (2) if and only if

$$
g(\lambda-0) \sim g(\lambda), \quad \lambda \rightarrow+\infty .
$$

Theorem 2. Let $\left\{f_{n}\right\} \in S^{\infty}$ and $g$ be a function in the class $F^{\infty}$ satisfying condition (7). If

(i) $f_{[g(\lambda)]} \sim \lambda, \lambda \rightarrow+\infty$,

(ii) $\lim _{\delta \rightarrow 1+0} \limsup _{n \rightarrow+\infty} \frac{f_{[n(1+\delta)]}}{f_{n}}=1$,

then for each sequence $\left\{\lambda_{n}\right\}$ satisfying (2), estimate (1) holds true.

And vice versa, if for each sequence $\left\{\lambda_{n}\right\}$ satisfying (2) estimate (1) holds, then $\left\{f_{n}\right\}$ and $g$ satisfy conditions (i) and (ii).

\section{Proof of Statements of Section 2}

3.1. Proof of Lemma 1. Since $\left\{f_{n}\right\}$ does not decrease, there exists a sequence of indices $\left\{n_{k}\right\}$ and an increasing sequence of $\left\{\nu_{k}\right\}$ such that

$$
f_{i}=\nu_{k}, \quad i=\overline{n_{k-1}+1, n_{k}} .
$$

We let $\lambda_{i}=f_{i}, i \in \mathbb{N}$. We have $N(\lambda)=n_{k-1}$ as $\lambda \in\left(\nu_{k-1}, \nu_{k}\right]$. According (2), for each $\varepsilon>0$ there exists $K_{\varepsilon} \in \mathbb{N}$ such that

$$
n_{k-1}(1-\varepsilon)<g(\lambda)<n_{k-1}(1+\varepsilon), \quad \lambda \in\left(\nu_{k-1}, \nu_{k}\right], \quad k \geqslant K_{\varepsilon} .
$$

Hence, since $g\left(f_{i}\right)=g\left(\nu_{k}\right)$ as $i=\overline{n_{k-1}+1, n_{k}}$, then

$$
g\left(f_{i}\right)<i(1+\varepsilon), \quad i=\overline{n_{k-1}+1, n_{k}} .
$$

We take another sequence satisfying (1): $\lambda_{i}=f_{i-1}$. Then $N(\lambda)=n_{k}$ as $\lambda \in\left(\nu_{k-1}, \nu_{k}\right]$ and therefore, by (2), for each $\varepsilon>0$ there exists a number $K_{\varepsilon} \in \mathbb{N}$ such that

$$
n_{k}(1-\varepsilon)<g(\lambda)<n_{k}(1+\varepsilon), \quad \lambda \in\left(\nu_{k-1}, \nu_{k}\right], \quad k \geqslant K_{\varepsilon} .
$$

Hence, since

$$
f_{i}=\nu_{k}, \quad i=\overline{n_{k-1}+1, n_{k}}
$$

then

$$
g\left(f_{i}\right)>n_{k}(1-\varepsilon) \text { for all } i=\overline{n_{k-1}+1, n_{k}}, \quad k \geqslant K_{\varepsilon} .
$$

Together with (8), this imples (5). The proof is complete.

3.2. Proof of Theorem 1. Sufficience of (5) and (6). Assume that (5) and (6) hold and $\left\{\lambda_{n}\right\}$ is a non-decreasing sequence with asymptotics (1). According Theorem BKS, $g\left(\lambda_{n}\right) \sim g\left(f_{n}\right)$, $n \rightarrow \infty$, and by (5) this implies

$$
g\left(\lambda_{n}\right) \sim n, \quad n \rightarrow \infty .
$$

Let $\left\{n_{k}\right\}$ and $\left\{\mu_{k}\right\}$ be increasing sequences such that $\lambda_{i}=\mu_{k}, k=\overline{n_{k-1}+1, n_{k}}, n_{0}=0$. Then $N(\lambda)=n_{k}$ as $\lambda \in\left(\mu_{k}, \mu_{k+1}\right]$. It follows from (9) that for each $\varepsilon>0$ there exists $K_{\varepsilon} \in \mathbb{N}$ such that for all $k \geqslant K_{\varepsilon}$ the inequalities

$$
n_{k}(1-\varepsilon) \leqslant g\left(\mu_{k}\right), \quad g\left(\mu_{k+1}\right) \leqslant\left(n_{k}+1\right)(1+\varepsilon)
$$


hold true. Hence, for all $\lambda \in\left(\mu_{k}, \mu_{k+1}\right]$ we get:

$$
n_{k}(1-\varepsilon) \leqslant g(\lambda) \leqslant\left(n_{k}+1\right)(1+\varepsilon),
$$

and this is why

$$
1-\varepsilon \leqslant \frac{g(\lambda)}{N(\lambda)} \leqslant\left(1+\frac{1}{n_{k}}\right)(1+\varepsilon), \quad \lambda>\mu_{k}, \quad k \geqslant K_{\varepsilon} .
$$

This proves (2).

Necessity. Let for each sequence $\left\{\lambda_{n}\right\}$ satisfying (1), relation (2) holds. Then condition (5) follows Lemma 1 ,

Let us prove (6). We assume the opposite and we are going to construct a non-decreasing sequence $\left\{\lambda_{n}\right\}$ satisfying (1) but not satisfying (2).

By Theorem BKS, there exists two asymptotically equivalent sequences $\left\{x_{n}\right\}$ and $\left\{y_{n}\right\}$ tending to infinity, for which at least one of the inequalities hold:

$$
\limsup _{n \rightarrow \infty} \frac{g\left(y_{n}\right)}{g\left(x_{n}\right)}>1 \quad \text { or } \quad \limsup _{n \rightarrow \infty} \frac{g\left(y_{n}\right)}{g\left(x_{n}\right)}<1 .
$$

Suppose that the first inequality holds; the case, when the second inequality holds can be considered in the same way. Then there exist a sequence $\left\{n_{i}\right\}$ and numbers $\alpha>0$ and $I_{1} \in \mathbb{N}$, such that

$$
g\left(y_{n_{i}}\right)>(1+\alpha) g\left(x_{n_{i}}\right), \quad i>I_{1} .
$$

Let $y_{n_{i}}=x_{n_{i}}\left(1+\varepsilon_{i}\right)$. We have $\varepsilon_{i} \rightarrow+0, i \rightarrow \infty$. Let also

$$
\sigma_{i}=\max _{k \geqslant i}\left\{\varepsilon_{k}\right\}, \quad m_{i}=\min \left\{k: f_{k} \geqslant x_{n_{i}}\right\} .
$$

Then

and according (5),

$$
f_{m_{i}-1}<x_{n_{i}} \leqslant f_{m_{i}}
$$

We let

$$
g\left(x_{n_{i}}\right) \sim m_{i}, \quad i \rightarrow \infty
$$

$$
\lambda_{m_{i}}=f_{m_{i}}\left(1+\sigma_{i}\right) .
$$

We can assume that the sequence $\lambda_{m_{i}}$ increases since we can choose a subsequence if it is needed.

According 12 , there exists $I_{2} \in \mathbb{N}$ such that

$$
g\left(x_{n_{i}}\right)>\frac{m_{i}\left(1+\frac{\alpha}{2}\right)}{1+\alpha}, \quad i \geqslant I_{2} .
$$

Then, since $g\left(x_{n_{i}}\left(1+\sigma_{i}\right)\right) \geqslant g\left(y_{n_{i}}\right)$, by 10 and (11) we get

$$
g\left(\lambda_{m_{i}}\right) \geqslant\left(1+\frac{\alpha}{2}\right) m_{i}, \quad i \geqslant I_{3}
$$

where $I_{3}=\max \left\{I_{1}, I_{2}\right\}$.

Let us define $\lambda_{k}$ for other $k$. Assume that $m_{i+1}>m_{i}+1$ and denote

$$
d_{i}=\max \left\{k \in\left[m_{i}, m_{i+1}\right): \quad f_{k}<\frac{f_{m_{i}}\left(1+\sigma_{i}\right)}{1+\sigma_{i+1}}\right\} .
$$

It is clear that $m_{i} \leqslant d_{i}<m_{i+1}$. We define

$$
\lambda_{k}=\left\{\begin{aligned}
f_{m_{i}}\left(1+\sigma_{i}\right), & m_{i} \leqslant k \leqslant d_{i}, \\
f_{k}\left(1+\sigma_{i+1}\right), & d_{i}+1 \leqslant k \leqslant m_{i+1} .
\end{aligned}\right.
$$


Then

$$
\lambda_{k} \sim f_{k}, d_{i}+1 \leqslant k \leqslant m_{i+1}, \quad i \rightarrow \infty .
$$

Let us prove that estimate (15) holds also for $m_{i} \leqslant k \leqslant d_{i}, i \rightarrow \infty$. For such $k$ we have

$$
\lambda_{k}=f_{m_{i}}\left(1+\sigma_{i}\right) \leqslant f_{k}\left(1+\sigma_{i}\right) .
$$

If $k \leqslant d_{i}$, then

$$
f_{k}<\frac{\lambda_{k}}{\left(1+\sigma_{i+1}\right)}
$$

and this implies

$$
\lambda_{k}>f_{k}\left(1+\sigma_{i+1}\right) .
$$

It follows from (16) and (17) that (15) as $m_{i} \leqslant k \leqslant d_{i}, i \rightarrow \infty$.

Thus, we have constructed a sequence $\left\{\lambda_{k}\right\}$ satisfying (1). Let us show that estimate (2) fails for this sequence.

It follows from the definition of $N(\lambda)$ that $N\left(\lambda_{m_{i}}\right) \leqslant m_{i}-1$ and this is why by (14) we get

$$
g\left(\lambda_{m_{i}}\right)>\left(1+\frac{\alpha}{2}\right) N\left(\lambda_{m_{i}}\right) .
$$

This implies that sequence $\left\{\lambda_{k}\right\}$ does not satisfy (2). The proof is complete.

3.3. Proof of Lemma 2. Sufficiency of (7). We let

$$
s_{i}=\sup \{\lambda: g(\lambda)<i\} .
$$

Since $g \in F^{\infty}$, then $\left\{s_{n}\right\} \in S^{\infty}$. Moreover, if $s_{i}<s_{i+1}$, then

$$
i \leqslant g(\lambda)<i+1 \quad \text { for all } \quad \lambda \in\left(s_{i}, s_{i+1}\right) .
$$

Let $\left\{n_{k}\right\}$ and $\left\{\mu_{k}\right\}$ be increasing sequences such that

$$
s_{i}=\mu_{k}, \quad i=\overline{n_{k-1}, n_{k}-1} .
$$

By (19), for $i=n_{k}-1$ we have:

$$
n_{k}-1 \leqslant g(\lambda)<n_{k}, \quad \lambda \in\left(\mu_{k}, \mu_{k+1}\right) .
$$

Employing again (19) with $i=n_{k}$, we obtain:

$$
g\left(\mu_{k+1}\right)<n_{k}+1
$$

Hence, since

$$
N_{s}(\lambda):=\max \left\{i: s_{i}<\lambda\right\}=n_{k}-1, \quad \lambda \in\left(\mu_{k}, \mu_{k+1}\right],
$$

we get

$$
1 \leqslant \frac{g(\lambda)}{N_{s}(\lambda)}<1+\frac{1}{n_{k}-1}, \quad \lambda \in\left(\mu_{k}, \mu_{k+1}\right) .
$$

By (21) we have $g\left(\mu_{k+1}-0\right) \leqslant n_{k}$. According (7), this implies

$$
g\left(\mu_{k+1}\right) \leqslant n_{k}\left(1+\alpha_{k}\right), \quad \alpha_{k} \rightarrow+0, \quad k \rightarrow \infty .
$$

Therefore,

$$
1<\frac{g\left(\mu_{k+1}\right)}{N_{s}\left(\mu_{k+1}\right)} \leqslant 1+\frac{1+\alpha_{k}}{n_{k}-1} .
$$

By (24), this allows us to conclude that the sequence $\left\{s_{n}\right\}$ satisfies (2).

Necessity. We assume the opposite, namely, let for some $\delta>0$ and some increasing sequence $\left\{\lambda_{n}\right\} \in S^{\infty}$ the inequalities

$$
g\left(\lambda_{n}-0\right)<(1-\delta) g\left(\lambda_{n}\right)
$$


hold. Since the function $N(\lambda)$ is left continuous, for each $n$ there exists a point $\mu_{n}<\lambda_{n}$ such that

$$
N\left(\mu_{n}\right)>N\left(\lambda_{n}\right)-\frac{1}{n}
$$

On the other hand, by (2) we get

$$
N\left(\mu_{n}\right)=g\left(\mu_{n}\right)(1+o(1)), \quad n \rightarrow \infty .
$$

Combining estimates 25)-27), we obtain

$$
\limsup _{n \rightarrow \infty} \frac{N\left(\lambda_{n}\right)}{g\left(\lambda_{n}\right)} \leqslant 1-\delta,
$$

which contradicts (2). The proof is complete.

3.4. Proof of Theorem 2. Sufficiency of (i) and (ii). Let Conditions (i) and (ii) hold, that is, for each $\varepsilon>0$ there exist $\Lambda_{1}(\varepsilon)>0, \delta(\varepsilon)>0$ and $n(\varepsilon) \in \mathbb{N}$, obeying the condition

$$
\begin{aligned}
& (1-\varepsilon) f_{[g(\lambda)]}<\lambda<f_{[g(\lambda)]}(1+\varepsilon), \quad \lambda>\Lambda_{1}(\varepsilon), \\
& f_{[n(1+\delta)]}<(1+\varepsilon) f_{n}, \quad 0<\delta<\delta(\varepsilon), \quad n \geqslant n(\varepsilon) .
\end{aligned}
$$

Let $\left\{\lambda_{n}\right\}$ be an arbitrary non-decreasing sequence satisfying condition (2), that is, for each $\sigma>0$ there exists $\Lambda_{2}(\sigma)>0$ such that

$$
g(\lambda)(1-\sigma)<N(\lambda)<g(\lambda)(1+\sigma) \quad \text { for all } \quad \lambda>\Lambda_{2}(\sigma) .
$$

Let $\left\{n_{k}\right\}$ and $\left\{\mu_{k}\right\}$ be increasing sequences defined by formula (20) such that $\lambda_{i}=\mu_{k}, i=$ $\overline{n_{k-1}, n_{k}}, n_{0}=0$. Then $N(\lambda)=n_{k}, \lambda \in\left(\mu_{k}, \mu_{k+1}\right], k \in \mathbb{N}$.

Given an arbitrary $\varepsilon>0$ we choose $\delta_{\varepsilon} \in(0, \delta(\varepsilon))$, where $\delta(\varepsilon)$ is determined by (29), and we let

$$
\Lambda_{3}(\varepsilon)=\max \left\{\Lambda_{1}(\varepsilon), \Lambda_{2}\left(\sigma_{\varepsilon}\right)\right\}, \quad \sigma_{\varepsilon}=\frac{\delta_{\varepsilon}}{1+\delta_{\varepsilon}} .
$$

Let $n(\varepsilon)$ satisfies $(29)$ and $K_{1}(\varepsilon), K_{2}(\varepsilon) \in \mathbb{N}$ be such that

$$
\begin{aligned}
& \mu_{k}>\Lambda_{3}(\varepsilon), \quad k \geqslant K_{1}(\varepsilon), \\
& n_{k}>\max \left\{n(\varepsilon)\left(1+\delta_{\varepsilon}\right), \frac{\left(1+\delta_{\varepsilon}\right)^{2}}{\delta_{\varepsilon}^{2}}\right\}, \quad k \geqslant K_{2}(\varepsilon) .
\end{aligned}
$$

We let $K(\varepsilon)=\max \left\{K_{1}(\varepsilon), K_{2}(\varepsilon)\right\}$ and let us show that

$$
f_{n_{k}}(1-2 \varepsilon) \leqslant \mu_{k+1}<f_{n_{k}}(1+\varepsilon)^{2}
$$

for all $k \geqslant K(\varepsilon)$.

Let $k \geqslant K(\varepsilon)$. We choose $\lambda=\mu_{k+1}$ in (30) and in view of (23) we get:

$$
g\left(\mu_{k+1}\right)<n_{k}\left(1+\delta_{\varepsilon}\right) .
$$

According (28) and (29) this yields

$$
\mu_{k+1}<f_{\left[n_{k}\left(1+\delta_{\varepsilon}\right)\right]}(1+\varepsilon)<f_{n_{k}}(1+\varepsilon)^{2} .
$$

We are going to prove the first inequality in (31). Let $\lambda \in\left(\mu_{k+1}, \mu_{k+2}\right]$. Then $\lambda>\Lambda_{1}(\varepsilon)$ and according 28 we have

On the other hand, since

$$
\lambda>(1-\varepsilon) f_{[g(\lambda)]} .
$$

$$
\lambda>\Lambda_{2}\left(\sigma_{\varepsilon}\right), \quad \frac{1}{1+\sigma_{\varepsilon}}>\frac{1}{1+\delta_{\varepsilon}},
$$


by $(30)$ and $(23)$ the inequality

$$
g(\lambda)>\frac{n_{k+1}-1}{1+\sigma_{\varepsilon}} \geqslant \frac{n_{k}}{1+\sigma_{\varepsilon}}
$$

holds and therefore,

$$
[g(\lambda)]>\frac{n_{k}}{1+\sigma_{\varepsilon}}-1
$$

We have

$$
\frac{n_{k}}{1+\sigma_{\varepsilon}}=\frac{n_{k}}{1+\delta_{\varepsilon}}\left(1+\frac{\delta_{\varepsilon}^{2}}{1+2 \delta_{\varepsilon}}\right)>\frac{n_{k}}{1+\delta_{\varepsilon}}\left(1+\frac{\delta_{\varepsilon}^{2}}{\left(1+\delta_{\varepsilon}\right)^{2}}\right) \text {, }
$$

and taking into consideration that $k \geqslant K_{2}(\varepsilon)$ and hence $n_{k}>\frac{\left(1+\delta_{\varepsilon}\right)^{3}}{\delta_{\varepsilon}^{2}}$, we obtain

$$
[g(\lambda)]>\frac{n_{k}}{1+\delta_{\varepsilon}}
$$

Hence, employing the definition of $K_{2}(\varepsilon)$ once again, we conclude that $[g(\lambda)]>n(\varepsilon)$ and we can use (29) with $n=[g(\lambda)]$. Taking also into consideration (33), we obtain

$$
f_{[g(\lambda)]}>\frac{f_{n_{k}}}{1+\varepsilon} .
$$

By inequalities 32 and

$$
\frac{1-\varepsilon}{1+\varepsilon}>1-2 \varepsilon
$$

we conclude that

$$
\lambda>(1-2 \varepsilon) f_{n_{k}} \quad \text { for all } \quad \lambda \in\left(\mu_{k+1}, \mu_{k+2}\right] .
$$

This completes the proof of the first inequality in $(31)$.

Necessity. Let us prove (i). Since the sequence $\left\{s_{n}\right\}$ constructed in the proof of Lemma 2 satisfies estimate (2), estimate (1) holds for this sequence as well:

$$
f_{n}=s_{n}\left(1+\sigma_{n}\right), \quad \text { where } \sigma_{n} \rightarrow 0, \quad n \rightarrow \infty .
$$

According (21), the identity $[g(\lambda)]=n_{k}-1$ holds for all $\lambda \in\left(\mu_{k}, \mu_{k+1}\right)$. Since $s_{n_{k}-1}=\mu_{k}$, we have

$$
f_{[g(\lambda)]}=\mu_{k}\left(1+\beta_{k}\right), \quad \lambda \in\left(\mu_{k}, \mu_{k+1}\right),
$$

where $\beta_{k}=\sigma_{n_{k}-1} \rightarrow 0, k \rightarrow \infty$. Hence,

$$
\frac{f_{[g(\lambda)]}}{\lambda}<1+\beta_{k}, \quad \lambda \in\left(\mu_{k}, \mu_{k+1}\right) .
$$

It follows from 22 that $\left[g\left(\mu_{k+1}\right)\right] \leqslant n_{k}$ and therefore

$$
f_{\left[g\left(\mu_{k+1}\right)\right]} \leqslant f_{n_{k}}=s_{n_{k}}\left(1+\sigma_{n_{k}}\right) .
$$

According (23), the identity holds $s_{n_{k}}=\mu_{k+1}$, and this is why the latter identity with (34) give

$$
\frac{f_{[g(\lambda)]}}{\lambda} \leqslant 1+\gamma_{k}, \quad \lambda \in\left(\mu_{k}, \mu_{k+1}\right]
$$

where $\gamma_{k} \rightarrow 0, k \rightarrow \infty$.

Let us prove that

where $\delta_{k} \rightarrow 0, k \rightarrow \infty$.

$$
\frac{f_{[g(\lambda)]}}{\lambda} \geqslant 1+\delta_{k}, \quad \lambda \in\left(\mu_{k}, \mu_{k+1}\right]
$$

We introduce the sequence

$$
\nu_{i}=\sup \{\lambda: g(\lambda)<i+1\}, \quad N_{\nu}(\lambda)=\sum_{\nu_{i}<\lambda} 1 .
$$


Since $\nu_{i}=s_{i+1}$, where $\left\{s_{i}\right\}$ is defined in (18), then $N_{\nu}(\lambda)=N_{s}(\lambda)-1$, and hence, $N_{\nu}(\lambda) \sim g(\lambda)$, $\lambda \rightarrow+\infty$. Therefore, estimate (1) holds also for sequence $\left\{\nu_{i}\right\}$, that is,

$$
f_{n}=\nu_{n}\left(1+\varepsilon_{n}\right), \quad \varepsilon_{n} \rightarrow 0, \quad n \rightarrow \infty .
$$

According (23), we have $\nu_{i}=\mu_{k}, i=\overline{n_{k-1}-1, n_{k}-2}$, where $\left\{\mu_{k}\right\}$ and $\left\{n_{k}\right\}$ are the same as in (20). Therefore, since $\nu_{n_{k}-1}=\mu_{k+1}$, taking into consideration (21) and (37), for all $\lambda \in\left(\mu_{k}, \mu_{k+1}\right)$ we get

$$
f_{[g(\lambda)]}=f_{n_{k}-1}=\mu_{k+1}\left(1+\delta_{k}\right)>\lambda\left(1+\delta_{k}\right),
$$

where $\delta_{k}=\varepsilon_{n_{k}-1} \rightarrow 0, k \rightarrow \infty$. This implies (36) as $\lambda \in\left(\mu_{k}, \mu_{k+1}\right)$.

In order to prove (36) as $\lambda=\mu_{k+1}$ we observe that by the first inequality in (21), we have $g\left(\mu_{k+1}\right) \geqslant n_{k}-1$ and therefore

$$
f_{\left[g\left(\mu_{k+1}\right)\right]} \geqslant f_{n_{k}-1}=\mu_{k+1}\left(1+\delta_{k}\right) .
$$

Relations (35) and (36) imply (i).

Let us prove (ii). We assume the opposite and we are going to construct a sequence satisfying (2) and not satisfying (1).

Assume that there exist two increasing sequences of natural numbers $\left\{\nu_{k}\right\}$ and $\left\{m_{k}\right\}$ and a positive number $\alpha$ such that

$$
\begin{aligned}
& \nu_{k}=m_{k}\left(1+\delta_{k}\right), \quad \delta_{k} \rightarrow+0, \quad k \rightarrow+\infty, \\
& f_{\nu_{k}}=(1+\alpha) f_{m_{k}} .
\end{aligned}
$$

Choosing a subsequence if it is needed, we can suppose that

$$
g\left(s_{m_{k+1}}\right)>g\left(s_{\nu_{k}}\right)
$$

for each $k$, where $s_{i}$ were defined by formula (18).

We introduce a sequence $\left\{p_{i}\right\}: p_{i}=0$ as $1 \leqslant i<m_{1}$ and

$$
p_{i}=\left\{\begin{array}{ll}
s_{\nu_{k}}, & m_{k} \leqslant i<\nu_{k}, \\
s_{i}, & \nu_{k} \leqslant i<m_{k+1},
\end{array} \quad k=1,2, \ldots\right.
$$

By Statement (i)

$$
f_{[g(\lambda)]}=\lambda(1+\delta(\lambda)), \quad \delta(\lambda) \rightarrow 0, \quad \lambda \rightarrow+\infty,
$$

and according $(19)$, for each $\varepsilon>0$ the inequality $g\left(s_{\nu_{k}}+\varepsilon\right) \geqslant \nu_{k}$ holds and hence

$$
f_{\nu_{k}} \leqslant f_{\left[g\left(s_{\nu_{k}}+\varepsilon\right)\right]}=\left(s_{\nu_{k}}+\varepsilon\right)\left(1+\delta\left(s_{\nu_{k}}+\varepsilon\right)\right)=\lambda_{m_{k}}(1+o(1)), \quad k \rightarrow+\infty .
$$

In view of $(39)$, for some sufficiently large $K_{0}$ we have

$$
p_{m_{k}} \geqslant\left(1+\frac{\alpha}{2}\right) f_{m_{k}}, \quad k \geqslant K_{0} .
$$

This implies estimate (1) for sequence $\left\{p_{i}\right\}$.

We are going to prove that the function $N_{p}(\lambda)=\max \left\{i: p_{i}<\lambda\right\}$ satisfies estimate (2). Since according (40), $N_{p}(\lambda)=N_{s}(\lambda)$ on $\left(s_{\nu_{k}}, s_{m_{k}}\right]$ for each $k \in \mathbb{N}$ and as it was shown in the proof of Lemma 2, the function $N_{s}(\cdot)$ satisfies estimate (2), it is sufficient to check that

$$
N_{p}(\lambda) \sim g(\lambda), \quad \lambda \in\left(s_{m_{k}}, s_{\nu_{k}}\right]
$$

as $k \rightarrow+\infty$. In view of the definition $\left\{p_{i}\right\}$ we have

$$
N_{p}(\lambda)=\nu_{k} \quad \text { as } \quad \lambda \in\left(s_{m_{k}}, s_{\nu_{k}}\right] .
$$

On the other hand, according (19),

$$
m_{k} \leqslant g(\lambda)<\nu_{k}
$$


for all $\lambda \in\left(s_{m_{k}}, s_{\nu_{k}}\right)$. Hence, in view of $(38)$, we conclude that

$$
1<\frac{N_{p}(\lambda)}{g(\lambda)}<1+\delta_{k}, \quad \lambda \in\left(s_{m_{k}}, s_{\nu_{k}}\right)
$$

where $\delta_{k} \rightarrow+0, k \rightarrow+\infty$.

Since by $(43)$ the inequality

$$
m_{k} \leqslant g\left(s_{\nu_{k}}-0\right) \leqslant \nu_{k}
$$

holds, we employ condition (18) and we get:

$$
m_{k} \leqslant g\left(s_{\nu_{k}}\right) \leqslant \nu_{k}\left(1+\alpha_{k}\right), \quad \alpha_{k} \rightarrow+0, \quad k \rightarrow+\infty .
$$

Together with $(42)$ this gives

$$
\frac{1}{1+\alpha_{k}} \leqslant \frac{N_{p}(\lambda)}{g(\lambda)} \leqslant 1+\delta_{k} .
$$

By estimate (44) this implies (41). The proof is complete.

\section{Example: Sturm-Liouville operator on a Curve}

Let $\gamma$ be a curve with the parametrization:

$$
z=\gamma(x)=\int_{0}^{x} \rho(t) e^{i \alpha(t)} d t, \quad x \in[0,1], \quad \int_{0}^{1} \rho(t) e^{i \alpha(t)} d t=1,
$$

where $r$ and $\alpha$ are piecewise continuous functions, $\rho \geqslant \rho_{0}, \rho_{0}=$ const $>0, \alpha$ is non-decreasing and numbers $\alpha_{0}=\alpha(0), \alpha_{1}=\alpha(1)$ satisfy the inequalities:

$$
-\frac{\pi}{2}<\alpha_{0}<0<\alpha_{1}<\frac{\pi}{2}
$$

Let $A C(\gamma)$ and $L^{p}(\gamma)$ be respectively the sets of the absolutely continuous functions and of $p$-summable functions with respect to the arc measure $|d z|$ on $\gamma$. Let $q \in L^{1}(\gamma)$. We denote by $L$ an operator on the domain

$$
D(L)=\left\{y \in L^{2}(\gamma): y^{\prime} \in A C(\gamma),-y^{\prime \prime}+q y \in L^{2}(\gamma), y(0)=y(1)=0\right\}
$$

acting in the Hilbert space $L^{2}(\gamma)$ by the rule

$$
L y=-y^{\prime \prime}+q y \text {. }
$$

As in the case $\gamma=[0,1][13, \S 17]$, one can confirm that $L$ is a closed domain with a dense domain. It is known [28, Lm. 2] that the spectrum of the domain $L$ is discrete and except for finitely many points, it is located in the angle $\left\{\mu \in \mathbb{C}:-2 \alpha_{1} \leqslant \arg \lambda \leqslant-2 \alpha_{0}\right\}$. We denote by $\left\{\lambda_{k}^{2}\right\}_{k=1}^{\infty},-\frac{\pi}{2}<\arg \left(\lambda_{k}\right) \leqslant \frac{\pi}{2}$, the eigenvalues of $L$ taken counting their algebraic multiplicities in the order of ascending absolute values. Then $\lambda_{k}$ are the zeroes of the function $\Phi(\lambda)=\varphi(1, \lambda)$, where $\varphi(z, \lambda)$ is the solution of the equation

$$
-y^{\prime \prime}+q y=\lambda^{2} y
$$

satisfying the conditions $\varphi(0, \lambda)=0, \varphi^{\prime}(0, \lambda)=1$. This implies that if $\Omega$ is a domain enveloped by the curve $\gamma$ and the segment $[0,1], q(z)=Q(z), z \in \gamma$, where $Q$ is a function holomorphic in the domain $\Omega$ and continuous on its closure, then the spectrum of the operator $L$ has an asymptotics

$$
\lambda_{k} \sim \pi k, \quad k \rightarrow \infty
$$

In fact, estimate (46) is ensured by the existence of a function $p \in L^{1}(0,1)$ such that the function

$$
\widetilde{q}(z)= \begin{cases}q(z), & z \in \gamma, \\ p(z), & z \in(0,1)\end{cases}
$$


satisfies a trivial monodromy condition on a closed curve $\Gamma=\gamma \cup[0,1]$ : for all values of the parameter $\lambda$, each solution to (45) is single-valued on $\Gamma$. As it was shown in [29], the trivial monodromy condition on $\Gamma$ is equivalent to the vanishing $q=Q$ almost everywhere on $\Gamma$, where $Q$ is a function meromorphic in the domain $\Omega$, having finitely many poles $z_{1}, \ldots, z_{n}$, and obeying the conditions:

A) ${ }_{n}$ For some $m_{i} \in \mathbb{N}, \delta_{i}>0, i=\overline{1, n}$, the identity holds:

$$
Q(z)=\frac{m_{i}\left(m_{i}-1\right)}{\left(z-z_{i}\right)^{2}}+\sum_{k=0}^{m_{i}-1} c_{k}\left(z-z_{i}\right)^{2 k}+O\left(\left(z-z_{i}\right)^{2 m_{i}-1}\right), \quad\left|z-z_{i}\right|<\delta_{i},
$$

B) $)_{n}$ The function

$$
\widetilde{Q}(z)=Q(z)-\sum_{i=1}^{n} \frac{m_{i}\left(m_{i}-1\right)}{\left(z-z_{i}\right)^{2}}
$$

belongs to the Smirnov space $E_{1}(\Omega)$ [30, Ch. III, Sect. 7].

The aim of this section is to show, by means of Theorem 2, that estimate (46) holds also in case, when the function $Q$ has infinitely many poles $\left\{z_{i}\right\}_{i=1}^{\infty}$ in $\Omega$, which can accumulate only to the segment $[0,1]$, and that apart of natural conditions of form (47) and b), the satisfy also some additional condition on the behavior in the vicinities of the points 0 and 1 .

Theorem 3. Let $q=Q$ almost everywhere on $\Gamma$, where $Q$ is a function meromorphic in the domain $\Omega$ and having poles $\left\{z_{i}\right\}_{i=1}^{\infty}$ satisfying the conditions

$\mathrm{A})_{\infty}$ There exist sequences $\left\{m_{i}\right\},\left\{\delta_{i}\right\}, m_{i} \in \mathbb{N}, \delta_{i}>0, i \in \mathbb{N}$ such that estimate (47) is valid for each $i$;

$\mathrm{B})_{\infty}$ For each piece-wise smooth curve $\gamma_{n}$ containing no poles of $Q$ and enveloping several first of them, function (48) belongs to $E_{1}\left(\Omega_{n}\right)$, where $\Omega_{n}$ is the domain enveloped by $\gamma_{n}$;

$\mathrm{C})_{\infty}$ If

$$
z_{k}=\left|z_{k}\right| e^{i \beta_{0 k}}=1+\left|z_{k}-1\right| e^{i \beta_{1 k}}, \quad \text { where } \quad-\frac{\pi}{2}<\beta_{0 k}<0, \quad-\pi<\beta_{0 k}<-\frac{\pi}{2},
$$

then $\beta_{0 k} \rightarrow 0, \beta_{1 k} \rightarrow-\pi, k \rightarrow \infty$.

Then estimate (46) holds for the eigenvalues of the operator $L$.

Proof. We denote by $n(r, \zeta, \theta)$ the number of the eigenvalues $\lambda_{k}$ in the sector $\{\lambda:|\lambda|<r, \zeta<$ $\arg \mu \leqslant \theta\}$. According Theorem 3 in [23], under Conditions $\mathrm{A})_{\infty}$ and $\left.\mathrm{B}\right)_{\infty}$, the function

$$
\Delta(\theta)=\lim _{r \rightarrow+\infty} \frac{n(r,-\pi / 2, \theta)}{r}
$$

reads as

$$
\Delta(\theta)= \begin{cases}0, & \theta \in\left(-\frac{\pi}{2}, 0\right), \\ \frac{1}{\pi}, & \theta \in\left(0, \frac{\pi}{2}\right] .\end{cases}
$$

By Condition $\mathrm{C})_{\infty}$, there exists a sequence of positive numbers $\left\{\varepsilon_{n}\right\}$ such that $\lim _{n \rightarrow \infty} \varepsilon_{n}=0$ and a polyline $\gamma_{n}$ with vertices at the points $0, \frac{1}{2\left(1-i \tan \varepsilon_{n}\right)}$ and 1 contains no poles of $Q$, and the number of the poles of $Q$ below $\gamma_{n}$ is finite. Then it follows from Theorem 1 in [29] that the spectrum of the operator $L$ coincides with spectrum of the operator $L_{n}$ obtained from $L$ via replacing $\gamma$ by $\gamma_{n}$. But according [28, Lm. 2], except for finitely many points, the spectrum of the operator $L_{n}$ is located inside the angle $|\arg z|<2 \varepsilon_{n}$. Arguing as in the proof of Theorem 1 in [20], we construct a continuous on $[0 ; \infty)$ function $\sigma$ satisfying the conditions:

a) $\sigma(x) \rightarrow 0, x \rightarrow \infty$, 
b) the domain

$$
D_{\sigma}=\{\lambda=x+i y: x>0,|y| \leqslant x \sigma(x)\}
$$

contains all eigenvalues $\lambda_{k}$.

Therefore, $\arg \lambda_{k} \rightarrow 0, k \rightarrow \infty$. This is why, to prove estimate 46), it is sufficient to make sure that

$$
r_{k}:=\left|\lambda_{k}\right| \sim \pi k, \quad k \rightarrow \infty .
$$

Let $N(r)=\sum_{r_{k}<r} 1$. We have $N(r)=n\left(-\frac{\pi}{2}, \frac{\pi}{2}\right)$. According 499, this implies that

$$
N(r) \sim \frac{r}{\pi}, \quad r \rightarrow+\infty
$$

Applying Theorem 2 to the sequence $\left\{r_{k}\right\}$ with $g(r)=\frac{\pi}{r}$ and $f_{k}=\pi k$, we obtain (50).

\section{BIBLIOGRAPHY}

1. G.V. Rozenblum, M.Z. Solomyak, M.A. Shubin. Spectral theory of differential operators // Itogi Nauki i Tekhniki. Ser. Sovrem. Probl. Mat. Fund. Napr. Partial differential equations-7. 64, 5-242 (1989). (in Russian).

2. V.A. Marchenko. Sturm-Liouville operators and applications. Naukova Dumka, Kiev (1977). [Birkhäuser Verlag, Basel (1986).]

3. M. Reed, B. Simon. Methods of modern mathematical physics. 4. Academic Press, New York (1978).

4. L. Hörmander. The analysis of linear partial differential operators. III: Pseudo-differential operators. Springer-Verlag, Berlin (1985).

5. D.G. Vasil'ev. Asymptotics of the spectrum of a boundary value problem // Trudy Mosk. Mat. Obschestva. 49, 167-237 (1986). (in Russian).

6. H. Weyl. Das Asymptotische Verteilungsgesetz der Eigenwerte linearer partieller Differentialgleichungen // Mathematische Annalen. 71:4, 441-479 (1912).

7. M. Kac. Can one hear the shape of a drum? // Amer. Math. Monthly. 73:4, 1-23 (1966).

8. V.V. Buldygin, O.I. Klesov, J.G. Steinebach. Properties of a subclass of avakumovic functions and their generalized inverses // Ukr. Math. Jour. 54:2, 179-206 (2002).

9. V.V. Buldygin, O.I. Klesov, J.G. Steinebach. On some extensions of Karamata's theory and their applications // Publ. Inst. Math. Nouv. Ser. 80(94), 59-96 (2006).

10. J. Karamata. Sur un mode de croissance régulière des fonctions // Mathematica (Cluj). 4, 33-53 (1930).

11. E. Seneta. Regularly varying functions. Lect. Notes Math. 508. Springer-Verlag, Berlin (1976).

12. N.H. Bingham, C.M. Goldie, J.L. Teugels. Regular variation. Encyclopedia of mathematics and its applications. 27. Cambridge Univ. Press, Cambridge (1987).

13. M.A. Naimark. Linear differential operators. Fizmatlit, Moscow (1969). [Frederick Ungar Publishing Co., New York. Part I. (1967); Part II. (1968).]

14. M.V. Keldysh. On eigenvalues and eigenfunctions of some classes of non-self-adjoint equations // Doklady AN SSSR. 77:1, 11-14 (1951). (in Russian).

15. A.A. Shkalikov. Perturbations of self-adjoint and normal operators with discrete spectrum // Uspekhi Matem. Nauk. 71(431):5, 113-174 (2016). [Russ. Math. Surveys. 71:5, 907-964 (2016).]

16. N. Dunford, J.T. Schwartz. Linear operators. Part II: Spectral theory. Self-adjoint operators in Hilbert space. Interscience Publ., New York (1963).

17. A.S. Markus, V.I. Matsaev. Comparison theorems for spectra of linear operators and spectral asymptotics // Trudy Mosk. Mat. Obschestva. 45. 133-181 (1982).

18. E.B. Davies. Non-self-adjoint differential operators // Bull. London Math. Soc. 34:5, 513-532 (2002).

19. Kh.K. Ishkin. A localization criterion for the eigenvalues of a spectrally unstable operator // Dokl. AN. 429:3, 301-304 (2009). [Dokl. Math. 80:3, 829-832 (2009).] 
20. Kh.K. Ishkin. On the spectral instability of the Sturm-Liouville operator with a complex potential. // Diff. Uravn. 45:4, 480-495 (2009). [Diff. Eqs. 45:4, 494-509 (2009).]

21. Kh.K. Ishkin. Conditions for localization of the limit spectrum of a model operator associated with the OrrSommerfeld equation // Dokl. AN. 445:5, 506-509 (2012). [Doklady Math. 86:1, 549-552 (2012).]

22. Kh.K. Ishkin. On analytic properties of Weyl function of SturmLiouville operator with a decaying complex potential // Ufimskij Matem. Zhurn. 5:1, 36-55 (2013). [Ufa Math. J. 5:1, 36-55 (2013).]

23. Kh.K. Ishkin. A localization criterion for the spectrum of the SturmLiouville operator on a curve // Alg. Anal. 28:1, 52-88 (2016). [St. Petersburg Math. J. 28:1, 37-63 (2017).]

24. I.C. Gohberg, M.G. Krein. Introduction to the theory of linear nonselfadjoint operators. Nauka, Moscow (1965). [Amer. Math. Soc. Providence, RI (1969).]

25. V.I. Matsaev, Yu.A. Palant. On distribution of spectrum of polynomial operator pencil // Doklady AN Armyan. SSR. 17:5, 257-261 (1966). (in Russian).

26. V.V. Buldygin, O.I. Klesov, J.G. Steinebach. Some properties of asymptotic quasi-inverse functions and their applications I // Theor. Probab. Math. Statist. 70, 11-28 (2005).

27. Kh.K. Ishkin. Conditions of spectrum localization for operators not close to self-adjoint operators // Dokl. RAN. 479:5, 497-500 (2018). [Dokl. Math. 97:2, 170-173 (2018).]

28. Kh.K. Ishkin. Necessary conditions for the localization of the spectrum of the SturmLiouville problem on a curve // Matem. Zamet. 78:1, 72-84 (2005). [Math. Notes, 78:1, 64-75 (2005).]

29. Kh.K. Ishkin. On a trivial monodromy criterion for the SturmLiouville equation // Matem. Zamet. [Math. Notes. 94:4, 508-523 (2013).]

30. I.I. Privalov. Boundary properties of analytic functions. GITTL, Moscow-Leningrad (1950). (in Russian).

Khabir Kabirovich Ishkin,

Bashkir State University,

Zaki Validi str., 32,

450074, Ufa, Russia

E-mail: Ishkin62@mail.ru

Rustem Ildarovich Marvanov,

Bashkir State University,

Zaki Validi str., 32,

450074, Ufa, Russia

E-mail: rsmar1v@gmail.com 